\title{
El gasto en salud relacionado con la condición de discapacidad. Un análisis en población pobre de México.
}

\author{
José E Urquieta-Salomón, MC, ${ }^{(I)}$ José L Figueroa, Act, ${ }^{(1)}$ Bernardo Hernández-Prado, PhD. ${ }^{(1)}$
}

\author{
Urquieta-Salomón JE, Figueroa JL, Hernández-Prado B. \\ El gasto en salud relacionado con la condición de discapacidad. \\ Un análisis en población pobre de México. \\ Salud Publica Mex 2008;5:136-I46.
}

\section{Resumen}

Objetivo. Estimar la relación entre discapacidad y gasto en salud en hogares pobres urbanos de México. Material y métodos. Con la Encuesta de Evaluación Urbana 2002 del Programa Oportunidades se identificaron hogares donde había personas con discapacidad estructural o con alguna limitación de actividades por enfermedad, y se estimó el gasto en salud ambulatorio y hospitalario. Resultados. De 15314 hogares estudiados, $10.1 \%$ incluyó a personas con discapacidad estructural y $13.4 \%$ con limitación de actividades moderada o grave. La discapacidad estructural en el hogar no se asoció con un mayor gasto en salud. Hogares donde había personas con limitaciones graves o moderadas para realizar sus actividades gastaron $97 \%$ más en atención ambulatoria que los hogares sin personas incapacitadas. Los hogares más pobres gastan más en atención médica que los hogares menos pobres. Conclusiones. Los resultados indican que la incapacidad para desarrollar actividades diarias tiene un importante efecto sobre el gasto de bolsillo, sobre todo en los hogares más pobres.

Palabras clave: personas con discapacidad; gastos en salud; pobreza; México
Urquieta-Salomón JE, Figueroa JL, Hernández-Prado B.

Health expenditure related to disability:

A study with poor population in Mexico.

Salud Publica Mex 2008;5:136-146.

\section{Abstract}

Objective. To estimate the effect of disability and incapacity in health expenditure in poor households in Mexico. Material and Methods. This is an analysis of baseline survey of the Oportunidades evaluation. Households with siblings with structural disability or incapacity were identified, and health expenditure was estimated. Results. In 15314 households analyzed, $10.1 \%$ had a sibling with structural disability, and $13.4 \%$ with mild or severe incapacity. The presence of structural disability was not associated with a higher expenditure in health care. The presence of mild or severe incapacity was associated with $97 \%$ higher expenditure in ambulatory care compared with households without incapacity. The poor households have higher health related expenditures. Conclusions. These results indicate that the incapacity to develop day to day activities has a significant impact on the out of pocket health expenditure. This impact is higher in poor households.

Key words: disabled persons; health expenditures; poverty; Mexico

(I) Dirección de Salud Reproductiva, Centro de Investigación en Salud Poblacional, Instituto Nacional de Salud Pública. Cuernavaca, Morelos, México.

Fecha de recibido: 4 de junio de 2007 • Fecha de aprobado: 8 de octubre de 2007

Solicitud de sobretiros: Mtro. José E. Urquieta. Centro de Investigación en Salud Poblacional, Instituto Nacional de Salud Pública. Av. Universidad 655, col. Santa María Ahuacatitlán. 62508 Cuernavaca, Morelos, México.

Correo electrónico: jurquieta@insp.mx 
L a discapacidad -entendida como la imposibilidad de realizar ciertas actividades o necesitar ayuda para hacerlas- es uno de los problemas de salud pública que adquirió relevancia en las décadas recientes. En México, los avances en diversos indicadores de desarrollo social y de salud han permitido extender la esperanza de vida y disminuir las tasas de mortalidad. ${ }^{1}$ No obstante, a menudo personas que en otras circunstancias habrían fallecido, sobreviven con secuelas físicas y/o mentales. Por tanto, cada vez un mayor número de personas en el mundo vive con algún tipo de discapacidad, con la consecuente carga económica para los sistemas de salud. ${ }^{2}$

En 1980 la Organización Mundial de la Salud (OMS) emitió la Clasificación Internacional de Deficiencias, Discapacidades y Minusvalías (CIDDM), ${ }^{3}$ la cual vincula las secuelas de la enfermedad con el funcionamiento físico, psicológico y la interacción social de las personas, más allá de la visión biomédica que se centra sólo en la enfermedad. En 2001, la revisión a la CIDDM dio origen a la Clasificación Internacional del Funcionamiento, la Discapacidad y la Salud (CIF), ${ }^{4,5}$ la cual concibe una relación sistémica entre el estado de salud, el trastorno o la enfermedad, y una dimensión amplia del funcionamiento de las personas que incluye un componente en el campo de la estructura y el funcionamiento corporal, y otro vinculado con la capacidad de las personas de participar y realizarse de manera normal en la sociedad. En este sentido, el término discapacidad abarca no sólo a la enfermedad, trastorno o anormalidad funcional de la persona, sino las limitaciones para llevar a cabo actividades en condiciones normales y las restricciones en la participación social.

La Organización de las Naciones Unidas (ONU) estima que hay alrededor de 600 millones de personas con discapacidad en el mundo; de éstas, dos terceras partes viven en países en desarrollo. ${ }^{6}$ Además, en 1990 se calculó que $98 \%$ de las personas con discapacidad en países en desarrollo carecía de servicio médico y de seguridad social. De acuerdo con datos recientes, en América Latina existen alrededor de 85 millones de personas con discapacidad, y más de un cuarto de la población total de la región se encuentra afectado de manera directa o indirecta por este fenómeno, ya sea por tratarse de familiares, amistades o miembros de la comunidad. ${ }^{7,8}$

Con base en la definición de la CIDDM, en México hay 1795000 personas con discapacidad, las cuales constituían $1.8 \%$ de la población en el año 2000.9,10 La discapacidad más frecuente es la motriz. Entre la población con alguna discapacidad, 75\% es desempleado, y aquellos que cuentan con empleo perciben un salario menor al de las personas no discapacitadas; además, la mayoría no tiene acceso a servicios de salud. ${ }^{11}$ Esto apoya la hipótesis de que hay una relación estrecha entre discapacidad y pobreza. La pobreza como factor contextual influyente en la discapacidad, y la discapacidad tendiente a profundizar la pobreza. ${ }^{12}$ Desde esta perspectiva, no es de extrañar que la mayoría de las personas con discapacidad viva en la pobreza.

$\mathrm{Al}$ respecto, resulta de suma importancia estudiar el costo de la discapacidad desde la perspectiva individual y social. Se ha documentado que la discapacidad representa una gran carga económica para los sistemas de seguridad social y para las personas. ${ }^{2}$ Los costos de la discapacidad se clasifican como directos e indirectos, según si incluyen los costos derivados de la atención médica o representan la pérdida de ganancias por la condición de discapacidad (pérdida de ingresos laborales, disminución de ingresos, costos de traslado, etc.). ${ }^{13}$

Ante la escasa literatura sobre este tema y la necesidad de estudiar la carga financiera ligada a la discapacidad en México, este estudio se propuso examinar el efecto de la discapacidad en el gasto de salud en hogares en condiciones de pobreza, con base en el supuesto de que los integrantes de un hogar con algún tipo de discapacidad no enfrentan sólo los costos que trae aparejada la misma, sino que implica una carga económica para la familia completa.

\section{Material y métodos}

Para este análisis se usó información de la Encuesta de Evaluación de los Hogares Urbanos 2002 (Encelurb 2002), que constituye la línea basal de una encuesta de seguimiento para la evaluación del impacto del Programa de Desarrollo Humano Oportunidades, un programa social dirigido a mejorar las condiciones de salud, educación y alimentación de familias que viven en extrema pobreza en México. La Encelurb 2002 abarcó hogares en áreas urbanas con una población de 50000 a un millón de habitantes. Se trató de un estudio cuasi experimental donde se seleccionó una muestra de manzanas (bloques de hogares) de manera probabilística, estratificada y por etapas en las zonas donde el programa empezó a operar en 2002, y mediante selección pareada por manzana en zonas donde el programa empezaría a operar en $2004 .{ }^{14}$

En una primera etapa se incluyeron todos los hogares que comprendían las manzanas seleccionadas y se clasificaron de acuerdo con su grado de pobreza o elegibilidad al programa. Los implementadores del programa determinaron el estatus de elegibilidad a partir de análisis discriminante con información socioeconómica de los miembros del hogar y su vivienda. La muestra final de la encuesta se conformó de aquellos hogares que manifestaron haber estado incorporados al programa en 
la primera etapa, y mediante un muestreo sistemático se seleccionó una muestra de hogares elegibles, casi elegibles y no elegibles. ${ }^{14}$ Para llevar a cabo este análisis se consideraron dos tipos: hogares elegibles y hogares no elegibles; en este último grupo se incluyó a los hogares casi elegibles que son aquellos que sobrepasaron apenas el punto mínimo de corte que define la elegibilidad a Oportunidades.

La Encelurb 2002 captó información sobre las características sociodemográficas y económicas de 17207 hogares y de las personas que los componen. Además de estos datos, en el análisis se usó el índice de marginación urbana 2000 publicado por el Consejo Nacional de Población (Conapo) ${ }^{15}$ agregado al ámbito de cada localidad, así como una serie de indicadores en el de entidad federativa que reflejan su desempeño en materia de salud. ${ }^{16}$

La unidad de análisis de este estudio fue el hogar, y se utilizó información proporcionada por la persona que contestó el cuestionario - la jefa de hogar en la mayoría de los casos- sobre la condición de discapacidad de todos sus miembros. Según la definición empleada por el INEGI, un individuo presenta lo que se denomina discapacidad estructural si tiene alguna limitación funcional para desempeñarse con normalidad; en términos más específicos, si manifiesta dificultad para moverse o caminar; dificultad para usar sus brazos o manos; es sordo o usa un aparato para oír; es mudo, ciego, o ve con sombras; si tiene algún retraso o deficiencia mental; o muestra tener otra limitación física o mental. Por otro lado, se identificó a todas aquellas personas del hogar mayores de cinco años sobre quienes se informó algún tipo de incapacidad por motivos de salud; esto es, personas que en las cuatro semanas previas a la encuesta pasaron días sin poder realizar sus actividades cotidianas: trabajar, ayudar a los quehaceres del hogar, ir a la escuela, cuidar a sus hijos, etc. Este tipo de discapacidad, a la que aquí se llama limitación de actividades por enfermedad, además de ser la más usual en la literatura, tiene importantes implicaciones para el análisis del gasto, ya que este tipo de discapacidad suele derivarse de eventos en salud imprevistos por los hogares.

Debido a la baja prevalencia esperada de hogares que tienen más de un miembro con discapacidad estructural, en este análisis se consideró un hogar con presencia de discapacidad estructural si cuando menos existe una persona con esta condición de salud. En relación con la limitación de actividades por enfermedad en mayores de cinco años, se estratificó por conveniencia la gravedad de la limitación según los días notificados como incapacitado: si registró tres o menos días, se consideró incapacidad leve; si la incapacidad tuvo una duración de cuatro a siete días, como moderada, y si la incapacidad duró más de una semana, como grave. Puesto que cabía la posibilidad de que un hogar informara de personas con varios tipos de limitaciones, se establecieron tres tipos de hogares: $a$ ) hogares sin discapacitados; $b$ ) hogares con cuando menos una persona con limitación leve de actividades y sin personas con limitaciones moderadas o graves, y c) hogares con personas con algún tipo de limitación de actividades, ya sea leve, moderada o grave. Además, en el análisis se tuvo en cuenta otras condiciones de salud, como la presencia en el hogar de personas con hipertensión arterial o diabetes. Si bien el control adecuado de estos padecimientos no implicaría caer en condición de discapacidad en el sentido de no limitar de forma sustancial el desempeño normal de las personas, repercute mucho en el gasto de los hogares por motivos de tratamiento.

El gasto relacionado con la salud se agregó al ámbito de cada hogar; esto quiere decir que se sumaron todos los gastos de bolsillo en salud informados para cada uno de los miembros del hogar. El gasto se dividió en dos componentes: como gasto ambulatorio se incluyeron los gastos en consultas médicas y medicinas en el último mes previo a la encuesta (hasta tres consultas), sin incluir hospitalización, y en el rubro gasto hospitalario se consideró el gasto por hospitalización (incluidas medicinas) notificado por el hogar en el último año previo a la encuesta.

En un primer análisis se exploró de manera descriptiva la prevalencia de discapacidad en el hogar y el patrón de gasto en servicios de salud por causas de discapacidad. Se puso especial énfasis en identificar el monto de estos gastos en los hogares elegibles y no elegibles al programa Oportunidades, en tanto interesaba saber si los hogares más pobres gastaban más en salud por causas de discapacidad que los menos pobres.

Para identificar los efectos en el gasto en salud por tener en el hogar miembros con alguna discapacidad estructural o limitación de actividades por enfermedad, se ajustaron modelos de regresión para cada uno de los dos tipos de gasto determinados: gasto ambulatorio y gasto hospitalario. El análisis se hizo para toda la muestra y para las submuestras de hogares elegibles y no elegibles a Oportunidades.

Debido a que parte importante de la población contaba con algún tipo de seguridad social o acceso a servicios asistenciales de salud, se encontró una proporción alta de nulo costo directo $(23 \%$ en gasto ambulatorio y $36 \%$ en gasto hospitalario), aun cuando manifestaron hacer uso de los servicios de salud. Para hacer una estimación consistente del modelo de regresión con la presencia de datos censurados, se calculó la ecuación de gasto sólo en los hogares que notificaron gasto positivo en salud, ajustada por la propensión de 
los hogares a realizar dicho gasto (modelo Heckit). Con esta especificación se contempla un posible sesgo de discriminación por no seleccionar de manera aleatoria a los hogares con gasto positivo. Es decir, pudiera haber factores no observables que afectaran, por un lado, la cantidad a gastar en servicios de salud y, por otro, la probabilidad de realizar dicho gasto. Si tal fuera el caso, se obtendrían estimadores sesgados e inconsistentes si no se corrigiera por dicha selectividad. ${ }^{17}$

El planteamiento general del modelo es: ${ }^{18}$

$$
\begin{aligned}
& y_{i}^{1}=X_{i} \beta_{l}+\varepsilon_{i}^{1} \\
& y_{i}^{*}=X_{i} \beta_{2}+Z_{i} \gamma+\varepsilon_{i}^{2}
\end{aligned}
$$

Donde $y_{i}^{1}$ es el logaritmo natural del gasto en salud (ambulatorio $\mathrm{u}$ hospitalario). $\mathrm{X}_{\mathrm{i}}$ representa los factores exógenos que afectan directamente el gasto de los hogares en salud, como la presencia de personas con discapacidad estructural o con alguna limitación de actividades por enfermedad, composición demográfica del hogar, nivel de ingreso al usar como proxy el gasto real mensual del hogar, tenencia de ciertos activos, condición de incorporación a Oportunidades y grado de marginación en su localidad. $Z_{i}$ son variables instrumentales que determinan la propensión del gasto en salud de los hogares, además de la tenencia de ciertos activos que podrían volverse líquidos para enfrentar compromisos de gasto; se incluyó una serie de indicadores que reflejan el desempeño de la entidad federativa en materia de salud, como el índice de justicia financiera (un indicador que mide el grado de equidad en el financiamiento del sistema de salud a través de su distribución proporcional a la capacidad de pago de los hogares), el gasto público en salud per cápita, o el gasto total en salud como proporción del PIB, que se espera repercutan en la eficiencia, calidad y accesibilidad de los servicios públicos de salud, pero no en el precio. Por su parte, $y^{*}$ es la propensión del hogar a gastar en salud y es una variable latente que toma los siguientes valores:

$$
\begin{aligned}
& T_{i}=1 \text { si } y^{*}>0 \\
& T_{i}=0 \text { de otra manera }
\end{aligned}
$$

La ecuación (2) denota el criterio de selectividad, es decir, $y_{i}^{1}$ sólo es observable si $T_{i}=1$. Como es posible que la $\operatorname{Cov}\left[\varepsilon_{i}^{1}, \varepsilon_{i}^{2}\right]$ sea diferente de cero, se estimó el sistema de ecuaciones de manera conjunta a través del método de máxima verosimilitud con corrección de los errores estándar por la posible correlación en cuanto a localidad. ${ }^{19}$

Las estimaciones con corrección por selectividad se hicieron para el gasto ambulatorio y hospitalario. Sin embargo, sólo se identificó correlación de significancia estadística entre las ecuaciones para el gasto ambulatorio, por lo que las estimaciones para el gasto hospitalario se hicieron por mínimos cuadrados ordinarios (MCO). Con los estimadores obtenidos se calculó el efecto proporcional promedio en el gasto en salud por tener en el hogar algún miembro con discapacidad estructural o con alguna limitación de actividades por enfermedad.

\section{Resultados}

Se obtuvo información de 15314 hogares. Se encontró que, por lo menos en $10.1 \%$ de éstos había una persona con discapacidad estructural; en $10.8 \%$, una con limitación leve de actividad, y en $13.4 \%$, una con limitación grave o moderada de actividades por enfermedad. Llama la atención que los hogares con jefes de hogar sin instrucción tengan mayor proporción de discapacitados, en cualquiera de sus tipos, que aquéllos con jefes de hogar que poseen mayor grado educativo (cuadro I).

Cuando se analizó el patrón de gasto de los hogares en servicios de salud ambulatorios $\mathrm{u}$ hospitalarios, se encontró que los hogares con personas limitadas de modo grave o moderado en sus actividades por enfermedad gastaron mucho más dinero en atención ambulatoria que aquéllos con personas con discapacidad estructural o con alguna limitación leve para realizar sus actividades (cuadro II). Por otro lado, la presencia de algún tipo de discapacidad en el hogar no afectó el gasto promedio en hospitalización notificado por los hogares.

Los hogares con al menos una persona derechohabiente a cualquier servicio médico, ya sea de seguridad social $\mathrm{u}$ otro tipo de seguro, tuvieron en promedio un gasto ambulatorio y hospitalario menor que los hogares sin esta prestación; destaca que la diferencia en el gasto promedio hospitalario es casi del doble. Los hogares con jefes de hogar sin instrucción reportaron en promedio mayor gasto en salud que aquéllos con jefes de hogar que poseen mayor instrucción. Los hogares más pobres o elegibles al programa Oportunidades tuvieron un mayor gasto hospitalario en comparación con los no elegibles. Asimismo, los hogares que vivían en localidades de alta y muy alta marginación informaron mayor gasto en salud respecto de los que vivían en localidades de mediana o baja marginación.

$\mathrm{Al}$ analizar la relación entre condición de pobreza, gasto en salud y presencia de discapacidad en el hogar, se encontró que los hogares elegibles al programa Oportunidades con presencia de cualquier tipo de discapacidad erogaron más en servicios ambulatorios y hospitalarios que los hogares no elegibles (cuadro III).

No sólo es importante saber cuánto gastan los hogares en atención para la salud, sino la carga financiera que esto significa para las familias. Una manera de medirlo 


\section{Cuadro I}

Presencia de discapacidad en hogares pobres urbanos. México, 2002

\begin{tabular}{|c|c|c|c|c|c|c|}
\hline & \multicolumn{3}{|c|}{$\begin{array}{l}\text { Presencia de discapacidad } \\
\text { estructural en el hogar }\end{array}$} & \multicolumn{3}{|c|}{$\begin{array}{l}\text { Presencia de personas con limitación } \\
\text { de actividades por enfermedad* }\end{array}$} \\
\hline & Total (N) & $\begin{array}{c}\text { Sin } \\
\text { discapacidad }\end{array}$ & $\begin{array}{l}\text { Con al menos una } \\
\text { persona con discapacidad }\end{array}$ & $\begin{array}{l}\text { Sin personas } \\
\text { con limitación }\end{array}$ & $\begin{array}{l}\text { Con limitación } \\
\text { leve }{ }^{\ddagger}\end{array}$ & $\begin{array}{l}\text { Con limitación } \\
\text { moderada o grave }\end{array}$ \\
\hline \multirow[t]{2}{*}{ Total } & 15314 & 13769 & I 545 & 11610 & 1653 & 2051 \\
\hline & $100 \%$ & $89.91 \%$ & $10.09 \%$ & $75.81 \%$ & $10.79 \%$ & $13.39 \%$ \\
\hline \multicolumn{7}{|l|}{ Estatus de elegibilidad } \\
\hline Elegible & 9690 & $90.31 \%$ & $9.69 \%$ & $76.17 \%$ & $10.57 \%$ & $13.26 \%$ \\
\hline No elegible & 5624 & $89.22 \%$ & $10.78 \%$ & $75.2 \%$ & $11.18 \%$ & $13.62 \%$ \\
\hline \multicolumn{7}{|c|}{ Escolaridad jefe del hogar } \\
\hline Sin escolaridad & 5770 & $87.61 \%$ & $12.39 \%$ & $74.69 \%$ & $10.5 \%$ & $14.9 \%$ \\
\hline Primaria & 4879 & $90.63 \%$ & $9.37 \%$ & $76.72 \%$ & $10.37 \%$ & $12.91 \%$ \\
\hline Secundaria y más & $4 I I 4$ & $92.51 \%$ & $7.49 \%$ & $76.11 \%$ & $12.11 \%$ & $11.79 \%$ \\
\hline \multicolumn{7}{|l|}{ Hogar derechohabiente } \\
\hline Sí & 3736 & $87.47 \%$ & $12.53 \%$ & $73.82 \%$ & $11.51 \%$ & $14.67 \%$ \\
\hline No & 11578 & $90.7 \%$ & $9.3 \%$ & $76.46 \%$ & $10.56 \%$ & $12.98 \%$ \\
\hline
\end{tabular}

es mediante el cálculo de la proporción del gasto en salud respecto del ingreso disponible de los hogares. Este indicador permite estimar el gasto catastrófico por motivos de salud y, en consecuencia, dimensionar el efecto en el bienestar de las familias. ${ }^{20} \mathrm{Si}$ se toma como proxy de ingreso el gasto total que realizan los hogares, en los hogares elegibles la proporción de gasto ambulatorio $\mathrm{u}$ hospitalario respecto de su gasto total supera en mucho al de los hogares no elegibles o menos pobres (cuadro III). Se concluyó que los hogares elegibles donde hay personas con alguna limitación moderada o grave para realizar sus actividades destinaron $26.9 \%$ de su gasto mensual en atención médica ambulatoria, proporción mayor que la informada por los hogares no elegibles.

Cuando se analizaron los factores asociados con el gasto ambulatorio $\mathrm{u}$ hospitalario de los hogares, se descubrió que la presencia de discapacidad estructural en el hogar no está significativamente asociada con el gasto en atención ambulatoria (cuadro IV). Para todos los hogares, la presencia de alguna persona con limitación leve por enfermedad para realizar sus actividades se vinculó con un incremento de $15.2 \%$ en el gasto, y la presencia de personas con limitación moderada o grave de sus actividades por enfermedad representó casi el doble del gasto ambulatorio (97.2\%) del que destinaron aquellos hogares sin personas con alguna limitación por enfermedad.

El análisis por tipo de hogar reveló que en los hogares elegibles la presencia de alguna persona con limitación leve para realizar sus actividades tuvo que ver con un aumento significativo en el gasto ambulatorio: $30 \%$, y de $106.8 \%$ si el hogar tuvo alguna persona con limitación moderada o grave para realizar sus actividades respecto de los hogares sin personas con alguna limitación. En los hogares no elegibles, la presencia de alguna persona con limitación leve no representó un incremento en el gasto en salud.

La presencia de discapacidad estructural en el hogar o de personas con alguna limitación de actividades por enfermedad no significó un alza relevante en el gasto hospitalario anual (cuadro V).

Los hogares con personas que sufren padecimientos -como hipertensión arterial- notificaron también mayor gasto en servicios de salud ambulatorios, y cuan- 


\section{Cuadro II}

\section{Gasto promedio en servicios de salud según Características socioeconómicas de los hogares*. México, 2002}

Caracteristica

Total

$\%$ con gasto positivo

Condición de discapacidad

$\mathrm{HH}$ sin discapacitados ${ }^{\#}$

$\%$ con gasto positivo

HH-discapacidad estructural ${ }^{2}$

$\%$ con gasto positivo

HH-limitación leve de actividad

$\%$ con gasto positivo

HH-limitación moderada o grave de actividad

$\%$ con gasto positivo

Hogar derechohabiente

Sí

$\%$ con gasto positivo

No

$\%$ con gasto positivo
Gasto en atención ambulatoria ${ }^{\ddagger}$

$354.54(841.80)$

77.0

287.23 (594.7I)

75.2

$297.28(515.55)$

74.6

$284.91(564.30)$

79.5

$567.14(1349.84)$

80.2
Gasto en atención hospitalaria ${ }^{\S}$

$2041.62(5737.86)$

64.0

$2004.80(5821.90)$

63.7

I601.71 (3285.63)

57.5

$2208.67(7329.30)$

63.7

$2176.86(5 \mid 43.74)$

65.8

Escolaridad del jefe del hogar

\begin{tabular}{lcc} 
Sin escolaridad & $384.53(1049.31)$ & $2176(5413.02)$ \\
\hline \% con gasto positivo & 77.1 & 68.5 \\
\hline Primaria & $347.00(713.80)$ & $1882.98(3993.93)$ \\
\hline$\%$ con gasto positivo & 78.7 & 65.9 \\
\hline Secundaria y más & $326.33(691.65)$ & $1907.81(5948.01)$ \\
\hline$\%$ con gasto positivo & 74.9 & 57.4
\end{tabular}

Estatus

\begin{tabular}{|c|c|c|}
\hline Elegible & $352.18(910.25)$ & $2209.57(6062.50)$ \\
\hline$\%$ con gasto positivo & 81.0 & 73.4 \\
\hline No elegible & 358.44 (723.24) & $177 \mid .78(5 \mid 65.66)$ \\
\hline$\%$ con gasto positivo & 77.1 & 48.9 \\
\hline \multicolumn{3}{|l|}{ Marginalidad } \\
\hline Media o baja & $335.80(685.18)$ & 1760.60 (4828.42) \\
\hline$\%$ con gasto positivo & 73.7 & 58.0 \\
\hline Alta & $364.36(732.02)$ & 2239.01 (6737.19) \\
\hline$\%$ con gasto positivo & 80.1 & 67.4 \\
\hline Muy alta & $369.92($ (II59.4I) & $2208.20(5368.30)$ \\
\hline$\%$ con gasto positivo & 77.4 & 68.9 \\
\hline \multicolumn{3}{|c|}{$\begin{array}{l}\text { * Gasto en pesos mexicanos e incluye a hogares con gasto cero } \\
\text { ‡ gasto en el último mes } \\
\text { § gasto en el último año } \\
\text { \# Son hogares que no tienen personas con discapacidad estructural ni presentan algún tipo de incapacidad o "discapacidad temporal” } \\
\text { \& Son hogares con al menos una persona con discapacidad estructural,y sin personas con algún tipo de limitación de actividad por enfermedad }\end{array}$} \\
\hline $\begin{array}{l}\text { HH: hogar } \\
\text { Desviación estándar en paréntesis }\end{array}$ & & \\
\hline
\end{tabular}

$239.55(6 \mid 2.98)$

51.2

399.80 (912.22)

87.08

278 (5459.59)

32.8

2419.04 (5835.53)

79.43 


\section{Cuadro III \\ Proporción y gasto PROMEdio de LOS hogaRes SEgún ESTATUS de elegibilidad a Oportunidades. México, 2002}

\begin{tabular}{|c|c|c|c|c|}
\hline & \multicolumn{2}{|c|}{ Gasto en atención ambulatoria* } & \multicolumn{2}{|c|}{ Gasto en atención hospitalaria* } \\
\hline & Elegible & No elegible & Elegible & No elegible \\
\hline \multirow[t]{2}{*}{ HH sin discapacitados } & 278.57 & 300.92 & 2120.11 & 1808.42 \\
\hline & $(585.75)$ & $(608.56)$ & $(6 \mid 48.65)$ & $(52 \mid 8.73)$ \\
\hline \multirow[t]{2}{*}{$\underline{\mathrm{HH} \text {-discapacidad estructural }}$} & 383.05 & 330.27 & 2776.86 & 919.90 \\
\hline & (975.01) & $(553.63)$ & (8904.49) & $(1749.69)$ \\
\hline \multirow[t]{2}{*}{ HH-limitación leve de actividad } & 288.61 & 279.39 & 2270.28 & 1346.20 \\
\hline & $(619.10)$ & $(47 I .25)$ & (839l.75) & $(5218.44)$ \\
\hline \multirow[t]{3}{*}{$\underline{\mathrm{HH} \text {-limitación moderada o grave de actividad }}$} & 572.62 & 558.55 & 2223.33 & 2108.31 \\
\hline & $(15 \mid 5.58)$ & $(1039.85)$ & $(4766.13)$ & $(5665.55)$ \\
\hline & \multicolumn{2}{|c|}{ Gasto ambulatorio/Gasto total mensual $\left.\right|^{\dagger}$} & \multicolumn{2}{|c|}{ Gasto ambulatorio/Gasto mensual en alimentos } \\
\hline$\underline{\mathrm{HH}}$ sin discapacitados & 14.22 & 12.47 & 26.52 & 24.30 \\
\hline HH-discapacidad estructural & 17.29 & 12.76 & 29.55 & 25.79 \\
\hline HH-limitación leve de actividad & 13.52 & 11.03 & 27.57 & 23.52 \\
\hline HH-limitación moderada o grave de actividad & 26.88 & 22.36 & 51.91 & 46.34 \\
\hline
\end{tabular}

HH: hogar

do había por lo menos un derechohabiente en el hogar se relacionó con un menor gasto promedio en salud; estos resultados fueron consistentes con lo esperado. Por último, la prueba de Wald -útil para verificar la independencia de las ecuaciones de gasto ambulatorio y la ecuación de probabilidad de gasto- revela que ninguna de las dos es independiente, es decir, hay decisiones comunes no observables que determinan la probabilidad de que un hogar gaste en atención médica y el monto de ese gasto; por tanto, el modelo empírico estimado es correcto.

\section{Discusión}

El presente trabajo buscó evaluar la relación entre el gasto en salud de los hogares y la presencia de algún tipo de discapacidad con base en la información contenida en la Encelurb 2002. La condición de discapacidad se clasificó en: discapacidad estructural (producto de alguna deficiencia física o mental del individuo) y discapaci- dad por enfermedad, aquí denominada limitación de actividades por enfermedad, la cual se dividió en leve, moderada o grave.

Esta diferenciación permitió concluir que la presencia de personas con alguna limitación de actividades por enfermedad tuvo un efecto importante en el gasto por motivos de salud de los hogares, sobre todo cuando las limitaciones fueron moderadas o graves, ya que representó casi el doble de gasto que desembolsaron los hogares sin personas discapacitadas, dato consistente con las conclusiones de otros estudios. 2,21,22 Este resultado apoya la hipótesis de que el mayor impacto por motivos de salud en el bienestar de los hogares se debe a shocks o imprevistos en salud, y que éstos son más grandes en hogares relativamente más pobres. Los resultados mostraron que los hogares más pobres, o elegibles al programa Oportunidades, con presencia de personas con algún tipo de discapacidad, destinaron mayor proporción de su gasto total en atención ambulatoria u hospitalaria que los hogares no elegibles. 


\section{Cuadro IV}

\section{Gasto en atención de salud ambulatoria*. Estimación por Máxima Verosimilitud con corrección POR SELECTIVIDAd SEgúN CONDICIÓN DE ElegibiLIDAd a Oportunidades. MéXico, 2002}

\begin{tabular}{|c|c|c|c|c|c|}
\hline \multirow[b]{3}{*}{$\begin{array}{c}\text { Gasto } \\
\text { ambulatorio }\end{array}$} & \multirow{3}{*}{$\begin{array}{c}\text { Ecuación } \\
\text { probabilidad } \\
\text { de gasto }\end{array}$} & \multicolumn{4}{|c|}{ Por condición de elegibilidad al Programa Oportunidades } \\
\hline & & \multirow[b]{2}{*}{$\begin{array}{c}\text { Gasto } \\
\text { ambulatorio }\end{array}$} & \multicolumn{2}{|c|}{ Ecuación } & \multirow{2}{*}{$\begin{array}{c}\text { Ecuación } \\
\text { probabilidad } \\
\text { de gasto }\end{array}$} \\
\hline & & & $\begin{array}{c}\text { probabilidad } \\
\text { de gasto }\end{array}$ & $\begin{array}{c}\text { Gasto } \\
\text { ambulatorio }\end{array}$ & \\
\hline & & Ele & gible & No e & gible \\
\hline
\end{tabular}

\begin{tabular}{|c|c|c|c|c|c|c|}
\hline \multicolumn{7}{|l|}{ Condición de salud en $\mathrm{HH}$} \\
\hline \multirow[t]{2}{*}{ HH-discapacidad estructural $(0=\sin$ discapacitados, I=sí) } & -0.008 & 0.097 & 0.072 & 0.077 & -0.113 & 0.116 \\
\hline & {$[0.14]$} & {$[2.34]^{\S}$} & {$[0.87]$} & {$[1.44]$} & {$[1.17]$} & {$[1.95]^{\ddagger}$} \\
\hline \multirow[t]{2}{*}{ HH-limitación leve de actividad ( $0=$ sin incapacitados, I=leve) } & 0.142 & 0.717 & 0.265 & 0.732 & 0.047 & 0.697 \\
\hline & {$[2.20]^{\S}$} & {$[17.84]^{\#}$} & {$[3.61]^{\#}$} & {$[15.83]^{\#}$} & {$[0.38]$} & {$[12.28]^{\#}$} \\
\hline \multirow[t]{2}{*}{ HH-limitación moderada o grave de actividad } & 0.679 & 0.918 & 0.727 & 0.938 & 0.717 & 0.905 \\
\hline & {$[8.59]^{\#}$} & {$[24.94]^{\#}$} & {$[9.21]^{\#}$} & {$[18.39]^{\#}$} & {$[5.11]^{\#}$} & {$[18.03]^{\#}$} \\
\hline \multirow[t]{2}{*}{ HH con algún adulto hipertenso ( $0=$ no, I=sí) } & 0.151 & 0.278 & 0.244 & 0.283 & 0.04 & 0.272 \\
\hline & {$[3.33]^{\#}$} & {$[9.40]^{\#}$} & {$[4.13]^{\#}$} & {$[7.70]^{\#}$} & {$[0.51]$} & {$[5.38]^{\#}$} \\
\hline \multirow[t]{2}{*}{$\mathrm{HH}$ con algún adulto diabético $(0=$ no, $I=$ sí $)$} & 0.046 & 0.064 & 0.068 & 0.036 & 0.006 & 0.087 \\
\hline & {$[0.88]$} & {$[1.73]^{\ddagger}$} & [0.86] & {$[0.70]$} & {$[0.08]$} & {$[1.64]$} \\
\hline \multirow[t]{2}{*}{ HH con mujer embarazada al momento encuesta $(0=$ no, I=sí) } & -0.025 & 0.21 & 0.017 & 0.208 & -0.079 & 0.232 \\
\hline & [0.40] & {$[6.08]^{\#}$} & [0.23] & {$[4.23]^{\#}$} & [0.74] & {$[3.24]^{\#}$} \\
\hline \multirow[t]{2}{*}{$\mathrm{HH}$ con mujer embarazada últimos 12 meses $(0=$ no, I=sí) } & 0.182 & 0.239 & 0.226 & 0.229 & 0.146 & 0.247 \\
\hline & {$[2.94]^{\#}$} & {$[6.40]^{\#}$} & {$[3.11]^{\#}$} & {$[5.01]^{\#}$} & {$[1.49]$} & {$[2.82]^{\#}$} \\
\hline Observaciones & 14538 & 14538 & 9173 & 9173 & 5365 & 5365 \\
\hline Prueba de Wald de independencia de eq. $($ rho $=0)$ & \multicolumn{2}{|c|}{$8.48^{\#}$} & \multicolumn{2}{|c|}{$13.44^{\#}$} & \multicolumn{2}{|c|}{$3.86^{\S}$} \\
\hline
\end{tabular}

* El gasto ambulatorio incluye el gasto mensual en servicios de salud ambulatorios y gasto en medicinas

‡ significativo a $10 \%$

$\S$ significativo a $5 \%$

\# significativo a $1 \%$

Estadísticos t robustos entre corchetes. EE ajustados por conjunto y por localidad $\mathrm{HH}:$ hogar

En las ecuaciones de gasto y probabilidad de gasto se incluye ajuste por presencia de mujeres en edad fértil, logaritmo natural y cuadrático del gasto total per cápita, número de personas en $\mathrm{HH}$, algún derechohabiente en el $\mathrm{HH}$, escolaridad del jefe del $\mathrm{HH}$, número de trabajadores en el $\mathrm{HH}$, edad y sexo del jefe del $\mathrm{HH}$, si en el $\mathrm{HH}$ hay niños de 0 a 5 años, niños de 6 a 12 años, jóvenes de 13 a $2 \mathrm{I}$ años, si en $\mathrm{HH}$ hay personas mayores a 60 años, estatus de incorporación a Oportunidades, si el HH sufrió algún siniestro natural en el último año, si el HH tiene aparatos eléctricos, TV,VHS, radio, refrigerador y animales de tiro. Además, por entidad federativa, se incluyó: gasto total en salud como proporción del PIB e índice de justicia financiera

En la ecuación de probabilidad de gasto se incluye ajuste por tipo de material de piso, techo y paredes de la vivienda, número de cuartos per cápita, si el HH tiene cocina exclusiva, agua en terreno, sanitario, drenaje, boiler, tinaco, estufa de gas; forma de recolección de basura; tipo de combustible para cocinar, y grado de marginalidad en la localidad (CONAPO 2000). Por entidad federativa, se incluyó tiempo de espera en unidades de primer nivel, gasto público como porcentaje del gasto total en salud, gasto público en salud per cápita, y logaritmo natural de la aportación estatal y federal a la salud En la ecuación de gasto se incluye ajuste por índice de marginalidad en la localidad (CONAPO 2000) 


\section{CuadroV \\ Gasto en atención hospitalaria*. Estimación por Mínimos Cuadrados Ordinarios SEgún Condición de elegibilidad a Oportunidades. México 2002}

\begin{tabular}{|c|c|c|c|}
\hline & Total & Elegible & No elegible \\
\hline \multicolumn{4}{|l|}{ Condición de salud en $\mathrm{HH}$} \\
\hline \multirow[t]{2}{*}{ HH-discapacidad estructural $(0=$ sin discapacitados, I = sí) } & -0.012 & 0.046 & -0.311 \\
\hline & {$[0.12]$} & {$[0.36]$} & {$[1.84]^{\ddagger}$} \\
\hline \multirow[t]{2}{*}{ HH-limitación leve de actividad ( 0 = sin incapacitados, I = leve $)$} & -0.154 & -0.096 & -0.109 \\
\hline & {$[1.57]$} & {$[0.89]$} & {$[0.49]$} \\
\hline \multirow[t]{2}{*}{ HH-limitación moderada o grave de actividad } & -0.059 & -0.067 & -0.018 \\
\hline & {$[1.04]$} & {$[0.84]$} & {$[0.12]$} \\
\hline \multirow[t]{2}{*}{$\mathrm{HH}$ con algún adulto hipertenso $(0=$ no, I = sí) } & 0.173 & 0.143 & 0.221 \\
\hline & {$[2.24]^{\S}$} & {$[1.90]^{\ddagger}$} & {$[\mathrm{l} .4 \mathrm{I}]$} \\
\hline \multirow[t]{2}{*}{ HH con algún adulto diabético $(0=$ no, I = sí) } & 0.04 & 0.149 & -0.018 \\
\hline & {$[0.38]$} & {$[1.27]$} & {$[0.09]$} \\
\hline \multirow[t]{2}{*}{$\mathrm{HH}$ con mujer embarazada al momento encuesta $(0=$ no, I = si $)$} & -0.084 & -0.069 & -0.161 \\
\hline & {$[0.70]$} & {$[0.42]$} & {$[0.77]$} \\
\hline HH con mujer embarazada últimos $I 2$ meses $(0=$ no, I = si $)$ & 0.218 & 0.193 & $0.24 I$ \\
\hline Observaciones & $|42|$ & 999 & 422 \\
\hline R-cuadrada & 0.14 & 0.15 & 0.22 \\
\hline \multicolumn{4}{|c|}{$\begin{array}{l}\text { * Corresponde al gasto en hospitalización en el último año en la encuesta e incluye medicinas y honorarios médicos } \\
\text { † significativo a } 10 \% \\
\S \text { significativo a } 5 \% \\
\# \text { significativo a } 1 \%\end{array}$} \\
\hline \multicolumn{4}{|c|}{$\begin{array}{l}\text { Estadísticos t robustos entre corchetes. EE ajustados por conjunto y por localidad } \\
\text { HH: hogar } \\
\text { Adicionalmente se controló por: presencia de mujeres en edad fértil, logaritmo natural y cuadrático del gasto total per cápita, número de personas en HH, } \mathrm{HH} \text {, } \\
\text { algún derechohabiente en el } \mathrm{HH} \text {, escolaridad del jefe del } \mathrm{HH} \text {, número de trabajadores en el } \mathrm{HH} \text {, edad y sexo del jefe del HH, si en el HH hay niños de } 0 \text { a } 5 \\
\text { años, niños de } 6 \text { a } 12 \text { años, jóvenes de } 13 \text { a } 2 \mathrm{I} \text { años, si en el HH hay personas mayores a } 60 \text { años, estatus de incorporación a Oportunidades, si el HH sufrió } \\
\text { algún siniestro natural en el último año; si el } \mathrm{HH} \text { cuenta con aparatos eléctricos,TV,VHS, radio, refrigerador y animales de tiro; tipo de material de piso, techo } \\
\text { y paredes de la vivienda, número de cuartos per cápita, y si el } \mathrm{HH} \text { tiene cocina exclusiva, agua en terreno, sanitario, drenaje, boiler, tinaco, estufa de gas; forma } \\
\text { de recolección de basura; tipo de combustible para cocinar, e índice de marginalidad en la localidad (CONAPO 2000). Por entidad federativa, se incluyó: } \\
\text { gasto total en salud como proporción del PIB, índice de justicia financiera, tiempo de espera en unidades de primer nivel, gasto público como porcentaje del } \\
\text { gasto total en salud, gasto público en salud per cápita, y logaritmo natural de la aportación estatal y federal a la salud }\end{array}$} \\
\hline
\end{tabular}

Es común que la presencia de discapacidad estructural en el hogar no repercuta en un mayor gasto ambulatorio u hospitalario, ya que, como se documentó, ${ }^{23}$ los hogares suelen suavizar su gasto por motivos de salud previsibles. Por supuesto que la discapacidad estructural tiene efectos negativos sobre el nivel de bienestar de los hogares, y su principal repercusión recae sobre la capacidad de las personas para acceder a mejores oportunidades educativas y laborales, lo que en última instancia redunda en su capacidad para generar ingresos.

En general, los hallazgos de este estudio son consistentes con los que arrojan otros estudios llevados a cabo con población mexicana. ${ }^{24-26}$ La presente inves- 
tigación confirma que los hogares más pobres son los más vulnerables, pues tienen fuertes restricciones para acceder a los esquemas de aseguramiento y son los que más gastan por motivos de salud. Si se aplica la definición más usada de gasto catastrófico, que se cumple si la proporción del gasto en salud respecto del ingreso neto disponible es mayor de 30\%, los hogares elegibles donde hay personas con alguna limitación moderada o grave de actividades poseen mayor riesgo de caer en gasto catastrófico. Por tanto, se prevé que la discapacidad producto de alguna enfermedad refleje una importante carga financiera en el bienestar de los hogares.

El estudio tiene algunas limitaciones que es necesario considerar al momento de interpretar los resultados. Es importante subrayar que los resultados que aquí se exponen no son extrapolables a toda la población pobre de México, ya que la selección de la muestra que se diseñó para la evaluación de un programa social resultó en un sobremuestreo de los hogares más pobres en áreas urbanas.

Contar con un diseño transversal impide identificar una secuencia causal entre discapacidad y gasto reportado en salud, ya que quizá la condición de discapacidad se habría presentado después de incurrir en el gasto en salud. Esta limitación puede explicar en parte que no se haya encontrado nexo entre el gasto en hospitalización, que fue medido por año, y la presencia en el hogar de personas con limitaciones de actividad, cuyo informe se refiere al último mes de la encuesta. Por otro lado, es posible que los hogares, al tener que enfrentar un gasto mayor por motivos de hospitalización, acudan a servicios asistenciales como los que proporciona la Secretaría de Salud, con lo que reducen su gasto directo de bolsillo. Otra importante limitación fue la imposibilidad de determinar las causas de enfermedad o padecimiento que originaron la limitación de actividades, lo que habría permitido explorar la relación entre ésta y la potencial discapacidad estructural.

Aun cuando los hallazgos indican que la presencia en el hogar de personas con alguna limitación de actividades por enfermedad está ligada a un mayor gasto en atención ambulatoria, dicha relación puede estar subestimada por no contemplar otros costos vinculados con el uso de los servicios de salud, como el costo de algún tipo de aseguramiento, el porcentaje de impuestos destinados al gasto público en salud, algunos costos indirectos como el que tiene que ver con el transporte $\mathrm{o}$, incluso, los días laborales perdidos por acudir a estos servicios.

Por último, los resultados de este estudio son importantes porque demuestran que la discapacidad estructural no afecta de manera importante el gasto que los hogares realizan en atención a la salud, y la incapa- cidad para realizar actividades cotidianas causada por enfermedad es la que tiene una relación significativa con un mayor gasto en salud; además, contribuyen a la escasa literatura en México para el entendimiento de los efectos de la discapacidad y otros factores en el gasto por motivos de salud. Los hallazgos confirman la situación de inequidad en la que se encuentran los hogares más pobres para enfrentar eventos imprevistos de salud, lo que alienta a estudiar en profundidad los efectos sobre el gasto en salud y el bienestar general de los hogares de iniciativas de atención a la pobreza como el programa de transferencias monetarias Oportunidades o del Seguro Popular. Asimismo, es pertinente insistir en la necesidad de instrumentar políticas que promuevan el cuidado de la salud tendientes a reducir la incidencia de cualquier tipo de discapacidad, así como a mejorar el acceso a los servicios de salud.

\section{Referencias}

I. Instituto Nacional de Estadística, Geografía e Informática. Indicadores sociodemográficos de México (1930-2000). México: INEGI, 200 I.

2. Rice D, LaPlante M. Medical expenditure for disability and disabling comorbidity.Am J Public Health 1992;82(5):739-74I.

3. Organización Mundial de la Salud. Clasificación Internacional de Deficiencias, Discapacidades y Minusvalías. Madrid: OMS, 1980.

4. Organización Mundial de la Salud. .Towards a common language for functioning, disability and health ICF. Geneve: OMS, 2002.

5. Egea-García C, Sarabia-Sánchez A. Clasificaciones de la OMS sobre discapacidad. Boletín 50 del Real Patronato sobre discapacidad. Madrid, 200I. [consultado: 2005 noviembre] Disponible en: http://www.rpd.es. 6. Organización de Naciones Unidas. Compendio de datos sobre los impedidos. Serie Y No. 4. New York: ONU, 1990.

7. International Disability Rights Monitor (IDRM). Monitoreo internacional de los derechos de las personas con discapacidad 2004. Informe regional de las Américas. Chicago: IDRM, 2004.

8. Organización Panamericana de la Salud. La salud en las Américas 2002;: : $173-224$.

9. XII Censo General de Población y Vivienda 2000. Estadísticas sociodemográficas. Disponible en: http://www.inegi.gob.mx.

10. Instituto Nacional de Estadística Geografía e Informática. Presencia del tema de discapacidad en la información estadística. Marco teóricometodológico. México: INEGI, 200I.

II. Grushka C, Demarco G. Disability pensions and social security reform. Analysis of the latin american experience. Washington, D.C.: World Bank, 2003.

12. World Health Organization. CBR:A strategy for rehabilitation, equalization of opportunities, poverty reduction and social inclusion of people with disabilities: joint position paper. [consultado: noviembre de 2005]. Disponible en: http://www.iol.org/public/english/employment/skills/ download/jointpaper.pdf.

13. Hernández J, Hernández I. Una aproximación a los costos indirectos de la discapacidad en Colombia. Rev Salud Pública 2005;7(2):I30-144.. 14. Behrman JR, Todd PE, Hernández-Prado B, Urquieta-Salomón JE, Attanasio O, Angelucci M, et al. Metodología de evaluación de Oportunidades en zonas urbanas 2002-2004 y rurales 1997-2003. En: Hernández-Prado B, Hernández-Ávila M, Urquieta-Salomón JE (eds.). 
Evaluación externa de impacto del Programa Oportunidades 2006. Tomo III, Documento metodológico. Cuernavaca, México: Instituto Nacional de Salud Pública, 2006.

15. Consejo Nacional de Población (Conapo). Índice de marginación urbana, 2000. [consultado: septiembre de 2005]. Disponible en: http:// www.conapo.gob.mx.

16. Secretaría de Salud. Salud México 2002. Información para la rendición de cuentas. México: Secretaría de Salud; 2003.

17. Heckman J. Sample selection bias as a specification error. Econometrica 1979;46:153-|6|.

18. Maddala GS. Limited dependent and qualitative variables in econometrics. New York: Cambridge University Press, 1983.

19. Johnston, DiNardo. Econometric methods. 4th. ed. McGraw-Hill International Editions, 1997

20. Secretaría de Salud. Síntesis ejecutiva: porcentaje de hogares con gastos catastróficos por motivos de salud 2000. México: SS, 2002

21.Altman B, Cooper P, Cunningham S. The case of disability in the family: impact on health care utilization and expenditures for nondisabled members. Milbank Q1999; 77(I): 39-75.
22. Dejong G, Palsbo S, Beatty P, Jones GC, Kroll T, Neri MT. The organization and financing of health services for persons with disabilities. Milbank Q 2002; 80(2): 26I-30I.

23. Gertler $P$, Gruber J. Insuring consumption against illness. Working Paper 6035. Cambridge, MA: NBER; 1997.

24. Sesma-Vázquez S, Pérez-Rico R, Sosa-Manzano CL, Gómez-Dantés O. Gastos catastróficos por motivos de salud en México: magnitud, distribución y determinantes. Salud Publica Mex 2005;47 (suplemento I): S37-S46.

25. Parker S, Wong R. Household income and health care expenditures in Mexico. Health Policy 1997;40:237-255.

26. Pérez-Rico R, Sesma-Vázquez S, Puentes-Rosas E. Gastos catastróficos por motivos de salud en México: Un estudio comparativo por grado de marginación. Salud Publica Mex 2005;47(Supl I):S47-S53. 